\title{
Optimal Location of Feedback Handler under Receiver Contention Schemes for Routing in Wireless Networks
}

\author{
Pai-Han Huang ${ }^{\mathrm{a}}$, Bhaskar Krishnamachari ${ }^{\mathrm{a}}$ \\ ${ }^{a}$ Dept. of Electrical Engineering - System, University of Southern California, Los Angeles, CA, \\ USA 90089-2560
}

\begin{abstract}
Due to the broadcast and error prone nature of wireless medium, novel routing mechanisms based on receiver contention have been proposed recently. The intuition of this strategy is, transmitters make routing decisions based on contentions of nodes that have successful reception. A remarkable advantage of receiver contention is the long average advancement of transmissions. To the best our knowledge, existing works utilizing receiver contention schemes are all based on a common assumption. That is, feedback packets sent by contending nodes are all destined to the transmitters. However, probability of reception is a function of distance. The longer the distance is, the lower the reception probability will be $\mathrm{s}^{5}$. According to this relation, we argue that transmitters may not be the best nodes to taking care of contention packets. In this paper, we consider uniformly distributed sensor networks, and propose the optimal locations, in terms of maximizing the expected advancement of each transmission, to place nodes which are responsible for handling feedback packets. We call these nodes feedback handlers. Based on the simulation results, placing the feedback handlers on the optimal locations can raise expected advancement up to about 30 percent, comparing to existing works.
\end{abstract}

Keywords: Receiver contention, receiver diversity, optimal location, sensor networks,

\section{INTRODUCTION}

Because of the broadcast and lossy nature of wireless medium, routing protocols designed for wired networks may be not suitable in wireless environment. Considering the uniqueness of sensor networks, many protocols and strategies have been proposed.

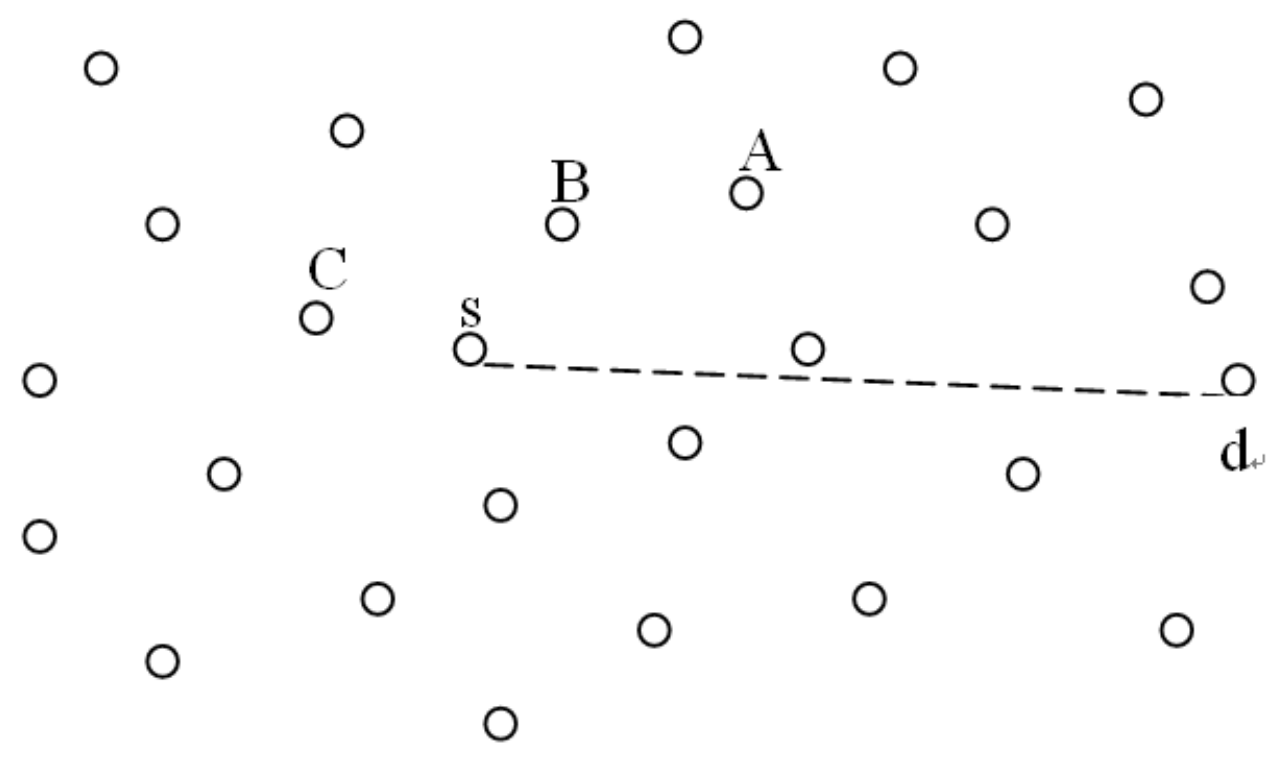

Fig. 1. A sample network. 
Because wireless transmission can be overheard by many nodes in the same neighborhood, failures of delivering packets to a specific node does not necessarily mean that no one can have successful reception. In short, there are two disadvantages existing in conventional routing protocols. First, because short and reliable links are preferred, the expected advancement for each transmission is limited, thus increasing hop counts from sources to destinations. Second, because only a set of nodes are selected to receive packets for each transmission, failure of reception in these nodes demands retransmissions for the same packets. Based on the above observations, a novel type of routing mechanism, called "receiver contention scheme," is proposed in recent works ${ }^{4,6}$. The intuition is, transmitters always adopt broadcasting while transmitting packets. Every node which has successful reception determines its eligibility to forwarding packets. If more than one is qualified, then the node which is closest to the destination will be chosen to be the next relay node. Consider an example in Figure 1. When the node S comes up with packets destined to the node D, it broadcasts these data to all neighboring nodes. Since the node $\mathrm{C}$ is located on the opposite side of the destination regarding the node $\mathrm{S}$, it is not a good choice to make the node $\mathrm{C}$ as the next hop because it will push the packets farther away from the destination. Therefore, if the node A, B, and C simultaneously receive these packets, only A and B are eligible to contend for the right of being the next hop node. In addition, because the node $\mathrm{A}$ is closer to the destination comparing with the node $\mathrm{B}$, it is reasonable to pick A as the next relay node for packets. Because of contentions of A and B for rights to relaying packets, this routing mechanism is named as "receiver contention." One noticeable advantage of receiver contention scheme is the long expected advancement for each transmission.

In order to determine which contention node is closer to the destination, protocols using receiver contention scheme must have the knowledge of geographical information of other nodes in the same network. Among existing protocols, two kinds of next-hop node assignment are proposed. One is explicit, and the other one is implicit. $\mathrm{GeRaF}^{6}$ is one representative work of explicit decision making protocol. In this kind of protocol, potential receivers, which overhear the transmissions, need to send contention packets to inform the transmitter. The transmitter then determines the next-hop node by explicitly sending a decision packet to that node. Because the transmitter only assigns one relay node for each transmission, there will no duplicate packets occur in $\mathrm{GeRaF}$ ExOR ${ }^{4}$, on the other hand, adopts implicit decision making policy. In ExOR, the transmitter needs to specify eligibility and priority ranking of nodes in packet header. When an eligible node, say A, overhears a transmission, it does not need to issue a contention packet. Instead, node A will set up a timer first, and the value of timer is related to its priority ranking as specified in the received packet header. If before the timer expires, A overhears some other node, which has higher priority than itself, forward the packet, then node A will drop the packet. Otherwise, node A starts forwarding the packet when its timer expires.

One potential problem of using implicit decision making policy is duplicate packet forwarding. Unlike the explicit approach, implicit strategy asks every potential receiver to make its own decision. A simple hidden terminal scenario can lead to unexpected duplicate forwarding problem, thus undermining the efficiency of protocols. ExOR adopts two approaches to overcome this problem. One is batch transmission, and the other one is embedding a batch map in every packet header. Intuitively, we would like a large batch and many receivers at the same time. However, these two techniques suffer from expensive overhead, and harm the total throughput. When a large number of batch size and receivers are chosen, the overhead is almost prohibition. In addition, optimal value of batch size and receiver number is still an open problem ${ }^{4}$.

Among the published works utilizing explicit decision making schemes, there exists a common assumption of dealing with contention packets. That is, all contention packets must be sent directly back to transmitters. Even in ExOR, it still relies on transmitters to decide which packets need to be re-transmitted. However, recent findings on wireless links ${ }^{5}$ suggest that, the assumption of symmetric channels is not suitable to practical situations. In addition, reception probability is a function of transmitting distance. The longer the distance is, the lower the reception probability will be. Based on this knowledge, we argue that, in order to maximize the expected advancement of each transmission, transmitters may be not the ideal ones to take care of contention packets. In this paper, we first present calculation of expected advancement considering a uniformly distributed network. Then, we try to find the optimal locations to place feedback handlers by formulating a optimization problem. We use numerical simulations to calculate the expected advancement comparing with original scheme. Based on the simulation results, placing the feedback handler on the optimal location can have 2 to 35 percent improvement on expected advancement, comparing with traditional feedback handling scheme.

This paper is organized as follows. We present the problem formulation in Section 2, including the method of calculation expected advancement, network topology, and link model. Based on the calculation results, we use numerical 
simulations to compare the improvement comparing with original scheme, present the results, observations, and discussions in Section 3. In Section 4, we conclude the contribution, and future directions of this paper.

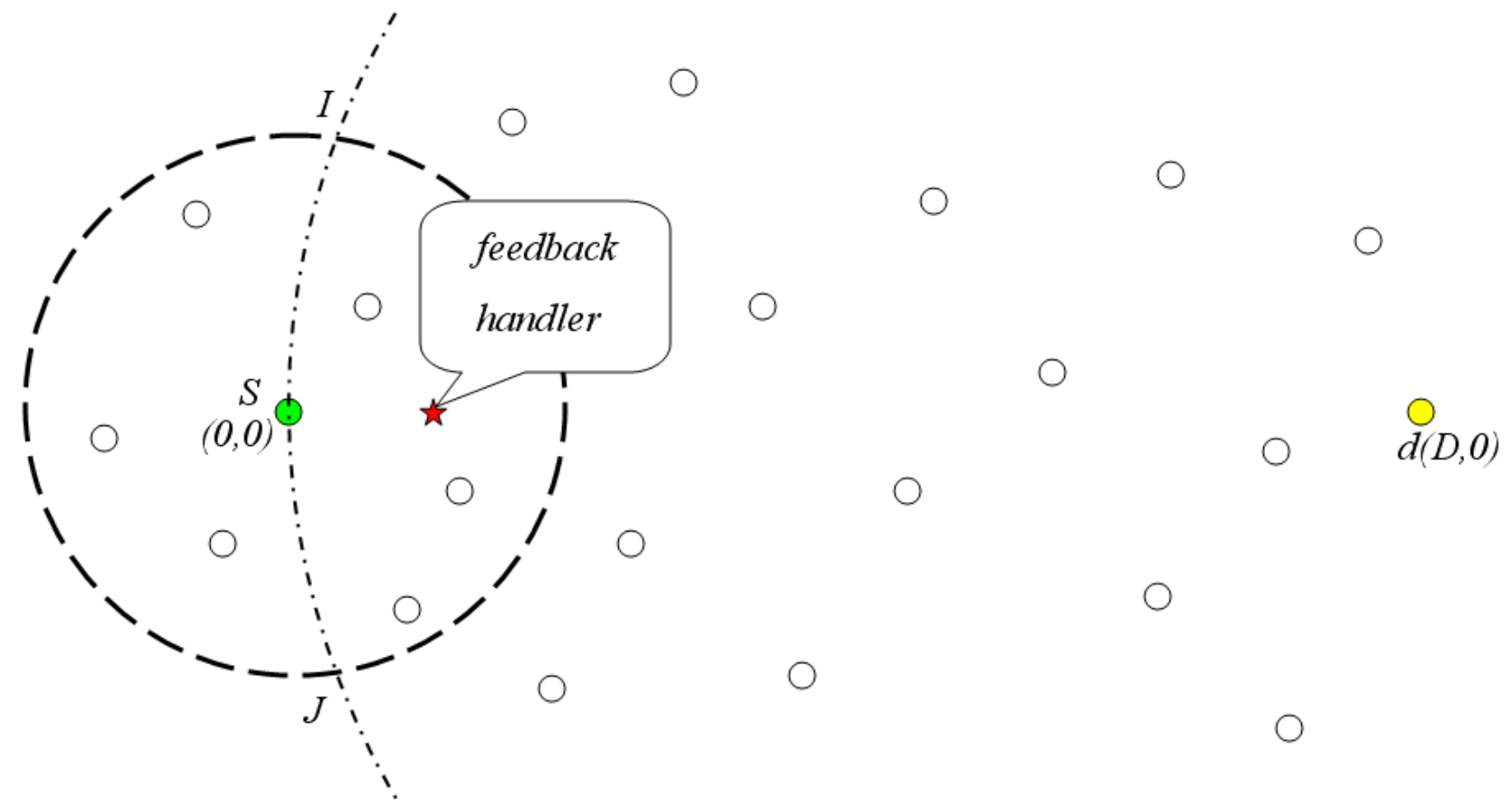

Fig. 2. Network topology.

\section{PROBLEM FORMULATION}

In order to find the optimal location to place feedback handler, we consider a uniformly distributed, 2D deployed sensor network with node density $\rho$ as plotted in Figure 2. There is one transmitter $s$ with transmission range $r$, and one destination $\mathrm{d}$, which is distance $\mathrm{d}$ away from the transmitter. Length of data packets and contention packets are $\mathrm{K}$ and $\mathrm{J}$, respectively. We assume a time-invariant channel, and every node within the transmission range uses the same reception probability model. In addition, we assume a proper MAC protocol is adopted. Therefore, no packet will be lost because of collisions. The area covered by a transmission is a disk with radius $\rho$, centered at the transmitter. The coordinates of $\mathrm{s}$ and $d$ is $(0,0)$ and $(D, 0)$, respectively. The coordinates of two intersection points, I and $J$, can be calculated as

$$
\begin{gathered}
I:\left(\frac{r^{2}}{2 D}, \frac{r}{2 D} \sqrt{4 D^{2}-r^{2}}\right) \\
J:\left(\frac{r^{2}}{2 D},-\frac{r}{2 D} \sqrt{4 D^{2}-r^{2}}\right)
\end{gathered}
$$

Advancement of a transmission, with data length $\mathrm{K}$, can be calculated as

$\operatorname{Exp}[$ advancement $]=\int_{-\frac{r}{2 D}}^{\frac{r}{2 D} \sqrt{4 D^{2}-r^{2}}} \int_{D-\sqrt{D^{2}-y^{2}}}^{\sqrt{r^{2}-y^{2}}} \rho \operatorname{Pr}_{1}(K) \operatorname{Pr}_{2,(m, n)}(1) d x d y$

where,

$\operatorname{Pr}_{1}(K)=$ Probability of data packet with length $K$ received by a node located at $(x, y)$

$\operatorname{Pr}_{2}(1)=$ Probability of contention packet with length 1 received by feedback handler located at $(m, n)$ 
According to [9], the expected packet reception rate can be calculated as:

$$
\operatorname{Exp}[\text { reception rate }]=\int_{\gamma_{0 e}}^{\gamma_{1 e}}\left(m_{e} \gamma+b_{e}\right) f\left(\gamma_{d b}, d\right) \delta_{\gamma d} B+Q\left(\frac{\gamma_{1 e}-\mu(d)}{\sigma}\right)
$$

The definitions of variables can be found in [9]. By using equation (3), we can get the value of $\operatorname{Pr}_{1}(K)$ and $\operatorname{Pr}_{2,(m, n)}(1)$. Therefore, our goal becomes finding the optimal value of $(m, n)$, such that:

$$
(m, n)=\underset{(m, n)}{\arg \max } \operatorname{Exp}[\text { advancement }]
$$

Because of the symmetry of the covered area, we can say the Y-axis coordinate of the optimal location of feedback handler is 0 . To simplify the problem, we normalize the value of $\mathrm{J}$ to be 1 during calculation. Moreover, because of sharp threshold of the packet reception rate, the expected packet reception rate can be farther simplified by a piece-wise linear approximation model without losing significant accuracy [9].

Although this problem can be modeled as an unconstrained optimization question, a closed form solution may not be achievable due to the absence of explicit double integral. In this paper, we use numerical analysis to find the optimal location of feedback handler.

Note that, the above formulation is versatile enough to include more other factors, which may influence the expected advancement. For example, if we need to take sleep scheduling policies into account, we may replace the parameter $\rho$, with $t \rho$, where the symbol $t$ represents the percentage of awaken nodes. If time-varying channel conditions need to be addressed, we can replace the equation (2) with a function of time. When the network is consisted of heterogeneous nodes, we can modify the packet reception model in equation (2) as well.

In this paper, we assume that there exists a routing protocol, such as [3], to avoid routing packets to dead end. Therefore, we only focus on maximizing expected packet advancement of each transmission.

\section{SIMULATION}

\subsection{Simulation Environment}

In order to verify the efficiency of placing feedback handler in the optimal location calculated by the above equations, we set up three experiments and compare their expected advancement with original feedback handling scheme. Throughout the whole simulations, we use the values in Table 1.

Table 1. Parameters.

\begin{tabular}{|c|c|}
\hline Parameter & Value \\
\hline Shadowing standard deviation $(\sigma)$ & 2.2 (except II) \\
\hline Path loss exponent $(\eta)$ & 3 \\
\hline Output power $(\mathrm{Pt})$ & -5 \\
\hline Noise floor $(\mathrm{Pn})$ & -105 \\
\hline Reference distance $\left(\mathrm{d}_{0}\right)$ & -55 \\
\hline Power decay for reference distance $\left(\mathrm{PL}\left(\mathrm{d}_{0}\right)\right)$ & 100 \\
\hline Data packet length $(\mathrm{K})$ & 1 \\
\hline Contention packet length $(f)$ & \\
\hline
\end{tabular}


In experiment $\mathrm{I}, \mathrm{K}$ is selected from 1 to 100 , and $\mathrm{d}$ varies from 10 to 1000 . We calculate the optimal location of the feedback handler, and compute the improvement of expected advancement comparing with original scheme.

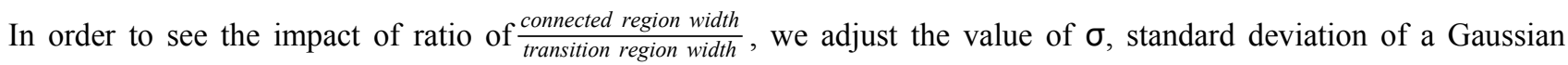
random variable in [9], from 2 to 3 in experiment II. Thus, the corresponding ratio of $\frac{\text { connected region width }}{\text { transition region width }}$ ranges from 1 to $\frac{1}{2}$.

In experiment III, we calculate the improvement when feedback handler is not placed on the optimal location. The intuition of this analysis is to provide an understanding of performance impact caused by location error. A detail descriptions of experiments is summarized in Table 2.

Table 2. Descriptions of Experiments.

\begin{tabular}{|c|c|}
\hline Experiment & Descriptions \\
\hline I & Change data packet length (K) and destination distance (D), and calculate the improvement. \\
\hline II & Change the ratio of $\frac{\text { connected region width }}{\text { transition region width by manipulating } \sigma \text { from } 2 \text { to } 3 .}$ \\
\hline III & Move the feedback handler in every place within the forwarding area. \\
\hline
\end{tabular}

\subsection{Simulation Results and Discussions}

The results of experiment I are depicted in Figure 3.

In experiment I, the expected advancement can be increased more than 27 percent by placing feedback handlers on the optimal location. We set the ratio of $\frac{\text { connected region width }}{\text { transition region width }}$ to be 1 , and the $\sigma$ in packet reception rate model to be 2 . That means, if the distance between a node and a transmitter is less than half of the transmission region, we almost for sure will have a successful reception. Figure 6 depicts the histogram of area covered by different radius of disk centered at transmitter and optimal feedback handler. It is noticeable that, around 44 percent forwarding area is within half of the transmission range to optimal location of feedback handler, comparing with 25 percent to the transmitter. Because the average distance of the whole forwarding region to the optimal feedback handler is significantly shorter than to the transmitter, we can benefit from placing feedback handlers on optimal locations. Figure 4 plots the optimal location of feedback handlers in experiment I. Note that, because of the symmetry of the forwarding region, the y-axis coordinates are always zero. Therefore, we only plot the $\mathrm{x}$-axis coordinates in this Figure. We can see that, when we fix the contention packet length to 1 and increase the data packet length $\mathrm{K}$, the optimal location moves towards the transmitter accordingly. This plot provides a reference when we plan deployments of feedback handlers. We do not plot the influence contributed by destination distance $\mathrm{d}$, because the impact of different $\mathrm{d}$ is less than 2 percent.

The results of experiment II are presented in Figure 5. In Figure 5 we can see that, while the ratio of $\frac{\text { connected region width }}{\text { transition region width }}$ decreases, the improvement of expected advancement decreases accordingly. The reason for this property comes from the packet reception model. In [9], the authors assume that every unit data reception process is independent with each other. The reception probability in transition region decreases geometrically with packet length. In addition, the smaller the ratio of $\frac{\text { connected region width }}{\text { transition region width }}$ is, the narrower the width of transition region will be. However, the improvement of expected advancement caused by optimal feedback handlers is mainly contributed by those nodes reside in transition region of transmitters. Therefore, it can be expected that we hardly can benefit a lot by placing feedback handlers on optimal locations, when the value of $\frac{\text { connected region width }}{\text { transition region width }}$ is small. Because the transition region becomes narrower when $\sigma$ decreases, we can expect the optimal location of feedback handler will be closer to the transmitter. This claim has been proved by Figure 7. Again, we only plot the x-axis coordinates because the y-axis coordinate is always zero.

In practical situations, precise placement of feedback handlers may not be possible. Therefore, it is useful to discuss the performance impact when feedback handlers are not placed exactly on the optimal location. Figure 8 plots the results of 
experiment III. The performance improvement demonstrates concavity regarding to the distance to the optimal location. In addition, the improvement is always positive, except the location around boundary of transmission range. Note that, even the location error is up to 30 percent of transmission radius $r$, we still can have 20 percent improvement. When precise placement is not possible, using nodes laid in the forwarding area to take care of contention packets, is still better than using transmitters themselves in terms of expected advancement of transmissions.

\section{CONCLUSIONS}

In this paper, we propose a novel idea to separate the responsibility of transmitter and feedback handler. Based on our simulation results, increase of expected advancement can be up to about $30 \%$ when the ratio of $\frac{\text { connected region width }}{\text { transition region width } 1}$ and $\mathrm{K}$ is 10 . In general, the improvement of expected advancement raises with increasing $\frac{\text { connected region width }}{\text { transition region width }}$ ratio, and decreasing data packet length $\mathrm{K}$. Even the feedback handler is not placed precisely on the optimal location, we still can expect more than 20 percent improvement when the location error is up to 30 percent of transmission radius. In addition, the model we present in this paper is highly versatile to further incorporate many other factors during network design stage. On the other hand, it is reasonable to say that the increase of communication overhead while implementing this new idea is low. Currently, we are working on a closed form approximation of the optimal location of feedback handlers with variables of packet length and $\sigma$. In the future, we plan to develop a control mechanism to utilize this idea and evaluate its performance by practical experiments.

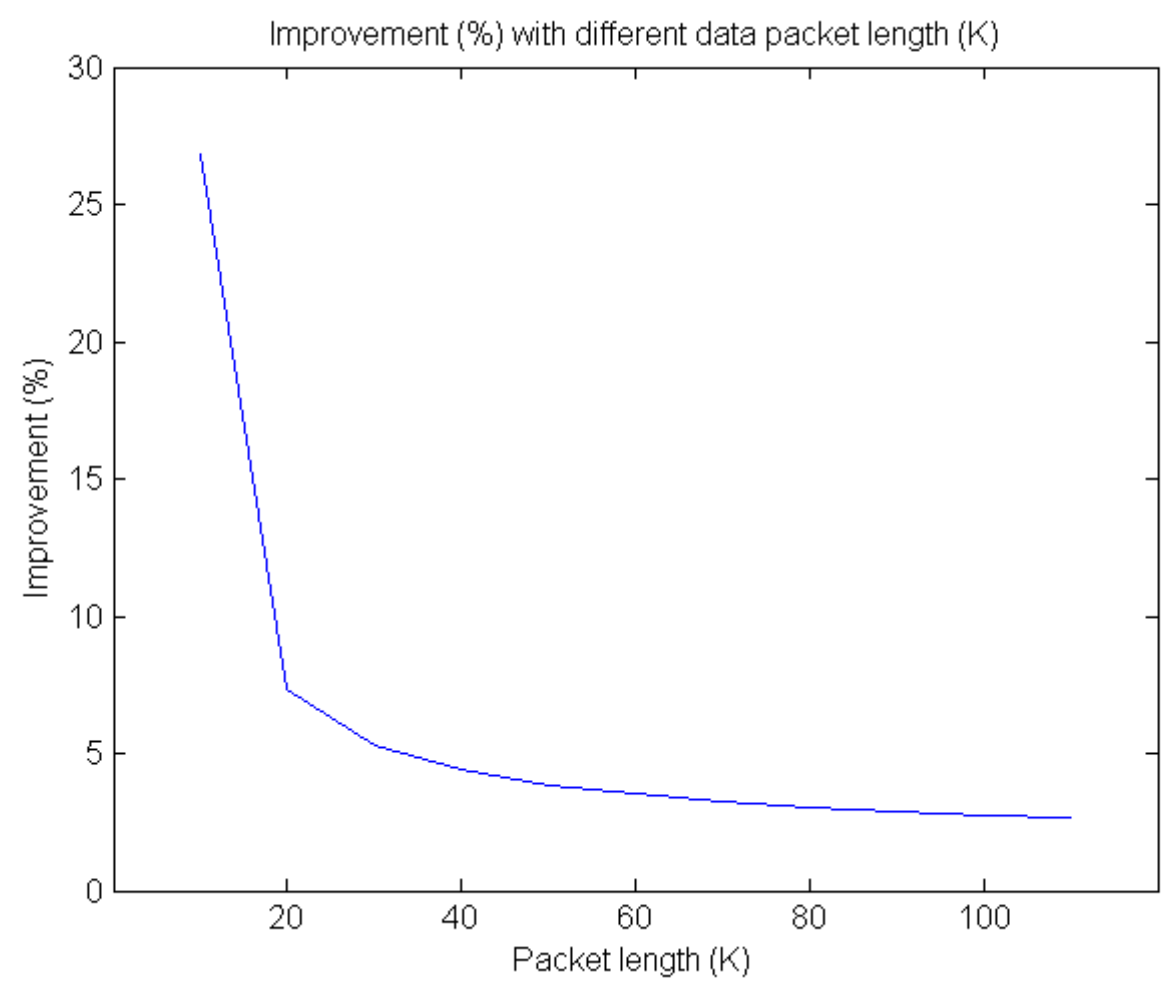

Fig. 3. Improvement under different packet length $(\mathrm{K}), \sigma$ is 2 in this plot. 


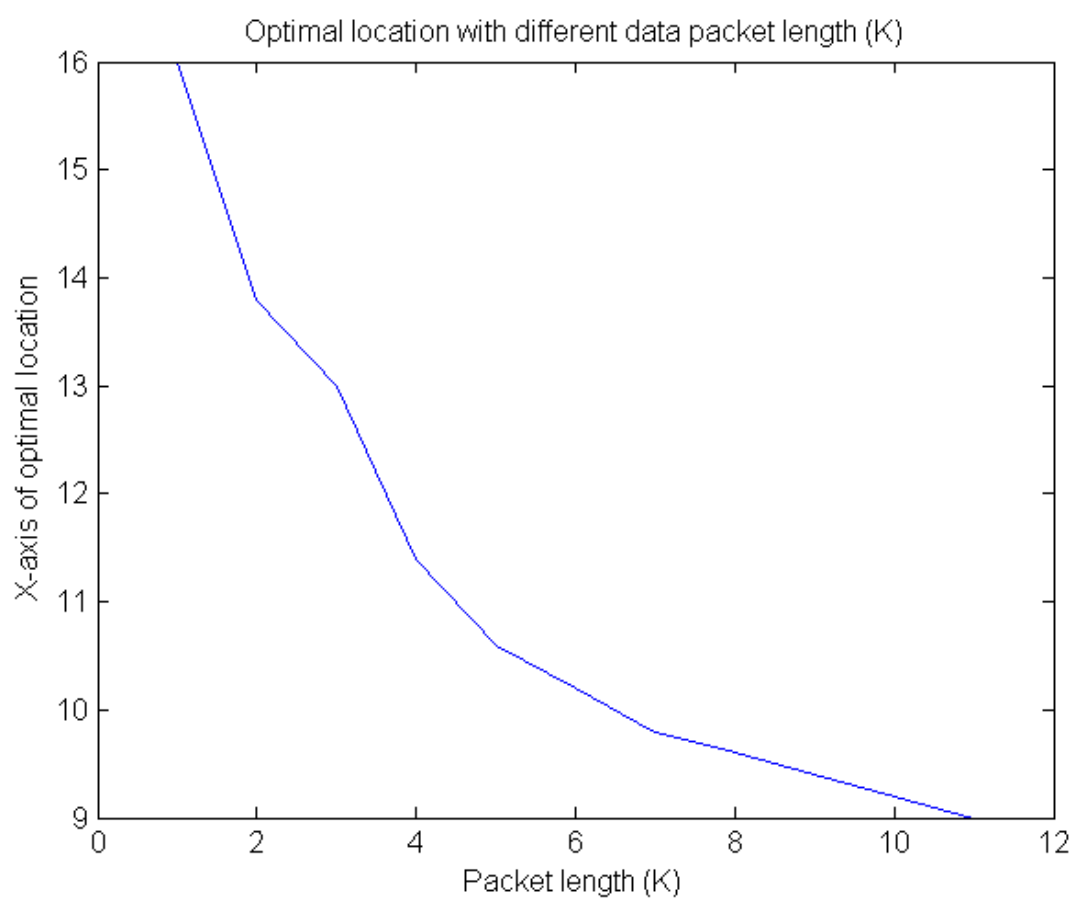

Fig. 4. Optimal location of the feedback handler under different packet length. Note that, because Y-axis coordinates are always 0 , we only plot the $\mathrm{x}$-axis coordinates of the optimal location in this Figure. $\sigma$ is 2 in this plot.

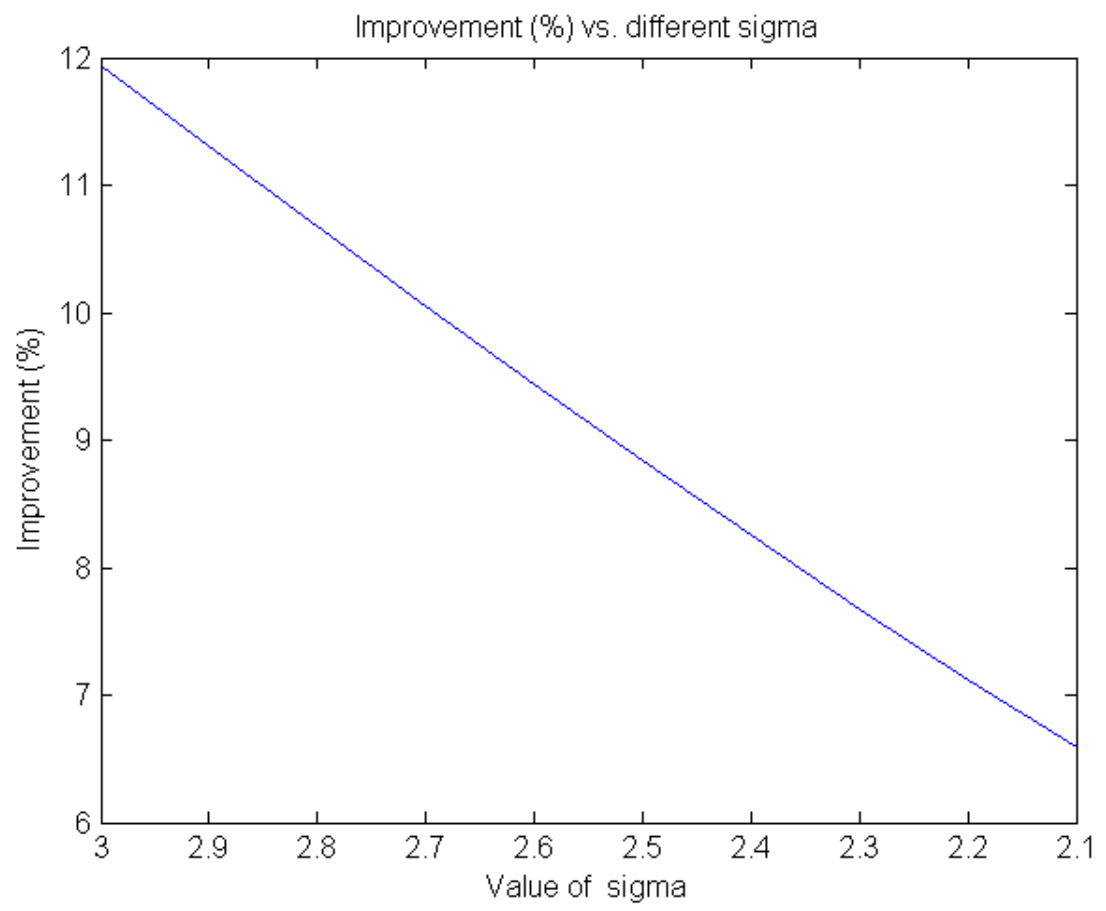

Fig. 5. Improvement under different ratio of $\frac{\text { connected region width }}{\text { transition region width }}$. 


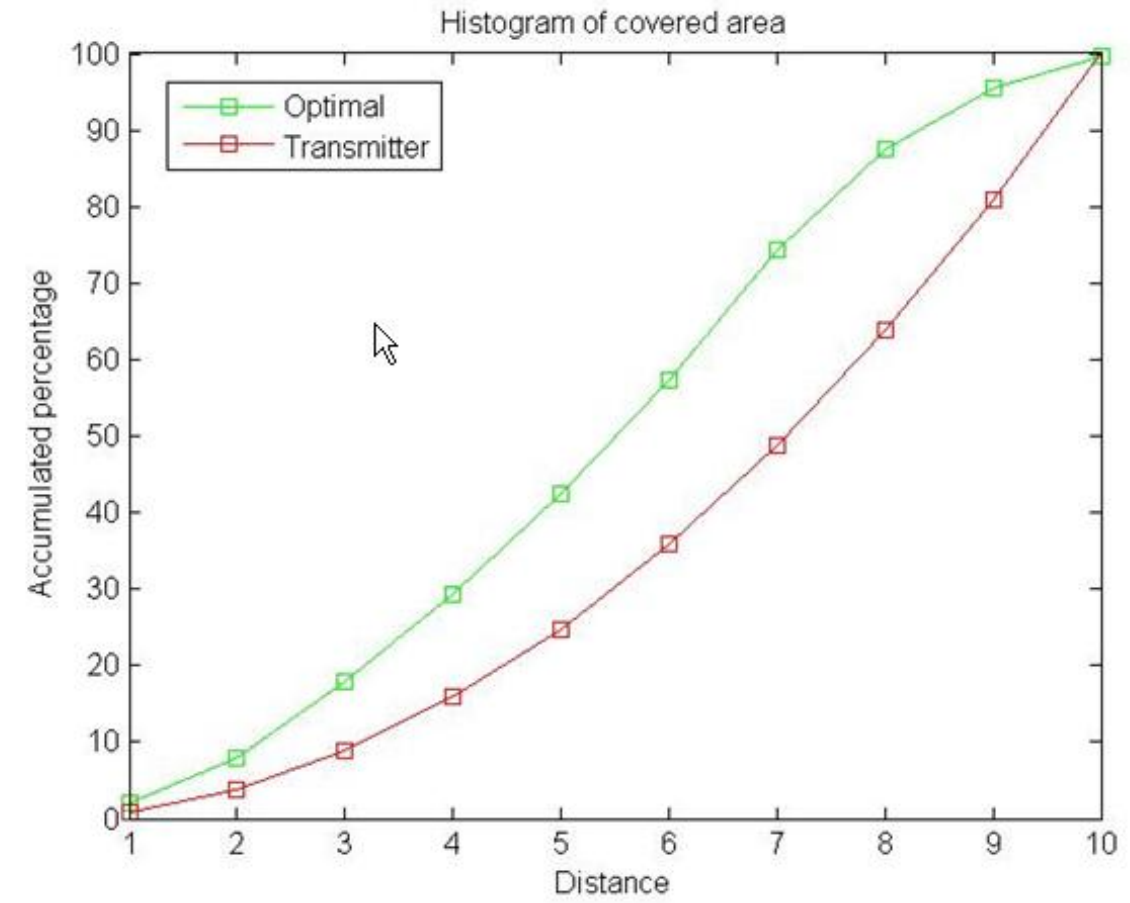

Fig. 6. Histogram of covered area under different distances to the transmitter and the optimal feedback handler location.

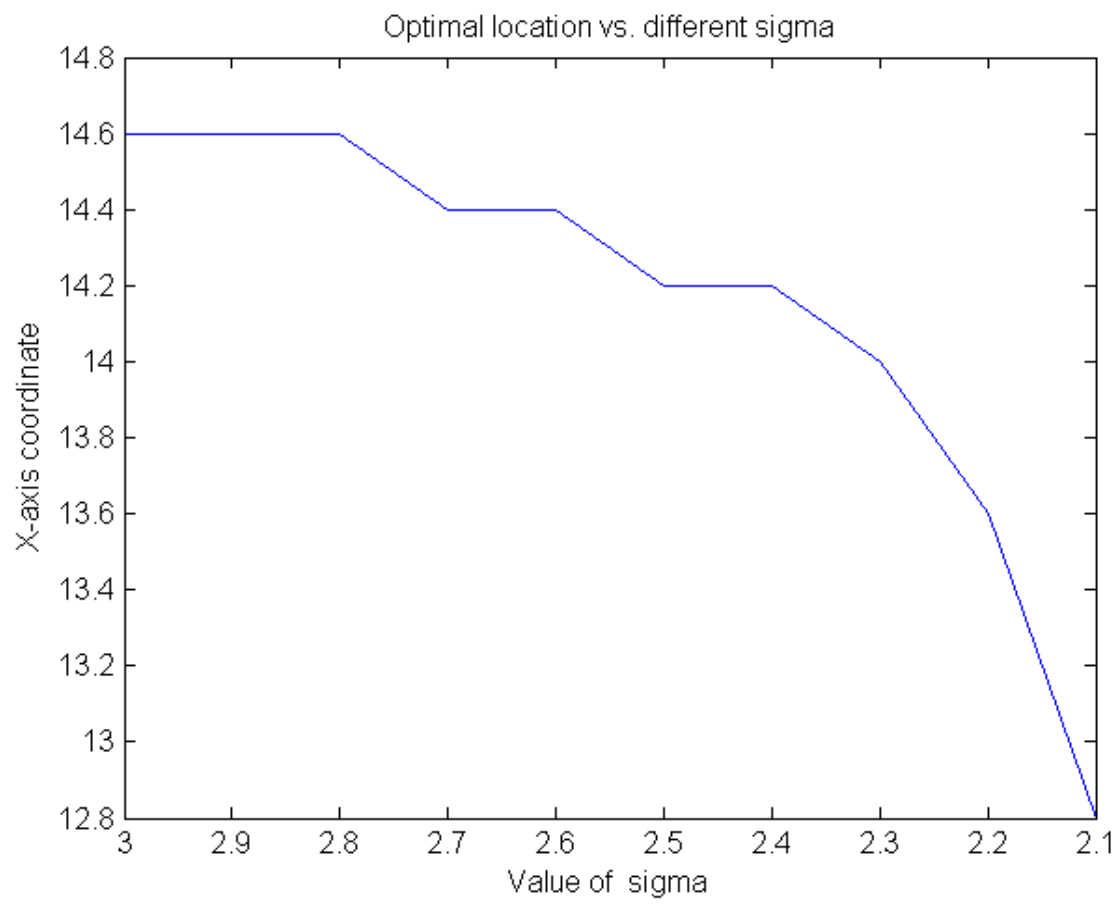

Fig. 7. Optimal location of feedback handler under different ratio of $\frac{\text { connected region width }}{\text { transition region width }}$. 


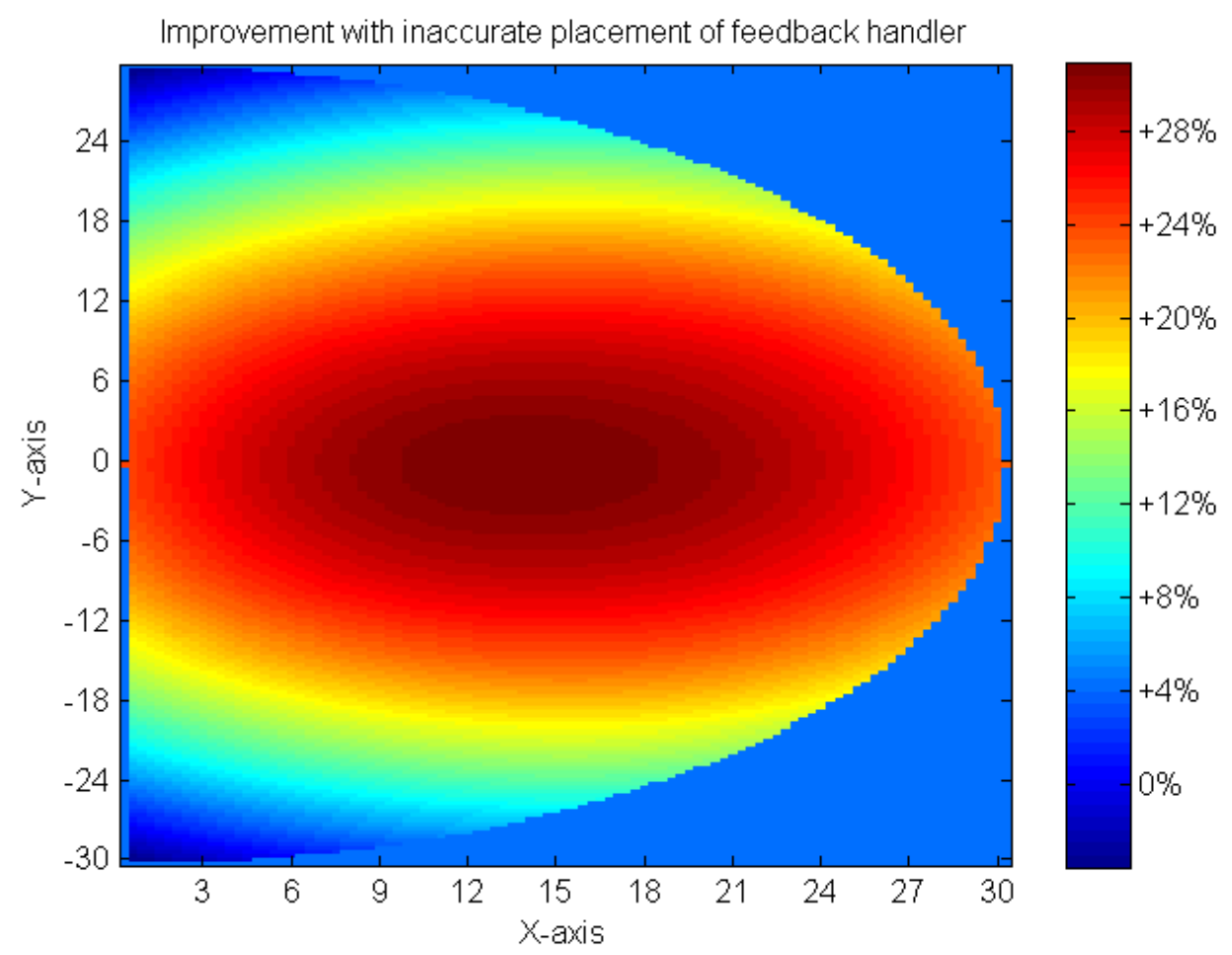

Fig. 8. Improvement of inaccurate placement of the feedback handler. $\sigma$ is 3 and $\mathrm{K}=10$ in this plot.

\section{ACKNOWLEDGMENTS}

This work was support in part by National Science Foundation through grants CNS-0347621.

Thanks Marco Zuniga for his constructive and valuable feedback on this work.

\section{REFERENCES}

1. C. Intanagonwiwat, R. Govindan, D. Estrin, J. Heidemann, and F. Silva, "Directed Diffusion for Wireless Sensor Networking." ACM/IEEE Transactions on Networking, 11, 1, February, 2002.

2. C. Intanagonwiwat, R. Govindan and D. Estrin, "Directed Diffusion: A Scalable and Robust Communication Paradigm for Sensor Networks." Proceedings of ACM MobiCom, August 2000.

3. B. Karp and H.-T. Kung, "GPSR: Greedy Perimeter Stateless Routing for Wireless Networks." Proceedings of ACM MobiCom, August 2000.

4. C. Biswas and R. Morris, "Opportunistic Routing in Multi-hop Wireless Networks." Computer Communication Review, 34, 1 (2004), 69-74.

5. M. Zuniga, B. Krishnamachari, "Analyzing the Transitional Region in Low Power Wireless Links", First IEEE International Conference on Sensor and Ad hoc Communications and Networks (SECON), Santa Clara, CA, October 2004.

6. M. Zorzi and R. Rao, "Geographic Random Forwarding (GeRaF) for Ad Hoc and Sensor Networks: Multihop Performance," IEEE Transactions on Mobile Computing 2003, vol.2, No.4, p337-348.

7. M. Zorzi, and R.R. Rao, "Geographic Random Forwarding (GeRaF) for Ad Hoc and Sensor Networks: Energy and Latency Performance." IEEE Trans. Mobile Computing, vol. 2, no. 4, Oct.-Dec. 2003. 
8. E. Khandani, E. Modiano, J. Abounadi and L. Zheng, "Reliability and Route Diversity in Wireless Networks." MIT LIDS Technical Report Number 2634, Nov. 2004.

9. M. Zuniga and B. Krishnamachari, "An Analysis of Unreliability and Asymmetry in Low-Power Wireless Links." First IEEE International Conference on Sensor and Ad hoc Communications and Networks (SECON), Santa Clara, CA, October 2004.

10. B. Krishnamachari, "Networking Wireless Sensors." Cambridge University Press, New York, 2006. 